\title{
Photoinitiated Thermal Degradation of Polymers II. Poly(methyl methacrylate)
}

\author{
Itaru Mita, ${ }^{*}$ Kazuhito Obata, and Kazuyuki HorIE* \\ Research Center for Advanced Science and Technology, \\ The University of Tokyo, 4-6-1, Komaba, Meguro-ku, Tokyo 153, Japan
}

(Received December 1, 1989)

\begin{abstract}
The thermal degradation of poly(methyl methacrylate) (PMMA) without chain-end double bonds was studied at $140-180^{\circ} \mathrm{C}$ by a photoinitiation method involving benzophenone derivatives. The temperature range of main-chain scission can be lowered from $270-330^{\circ} \mathrm{C}$, the usual thermal degradation condition for anionically prepared poly(methyl methacrylate), to the $140-180^{\circ} \mathrm{C}$ temperature range of the photoinitiated case. The formation of a PMMA on-chain radical by hydrogen abstraction by a triplet benzophenone derivative with low initiator efficiency $(f=0.02-0.03)$ is followed by its $\beta$-scission and depolymerization with a zip length of $790-3200$ at $140-180^{\circ} \mathrm{C}$ for PMMA with $M_{n 0}=8.4 \times 10^{5}$. A first-order termination mechanism for the polymer radical is suggested, based on the irradiation intensity dependence of the chain scission. The rate of weight loss during thermal degradation is independent of the molecular weight of PMMA, but the rate of main-chain scission increases with decreasing molecular weight, suggesting that a terminal alkyl radical produced by complete depolymerization undergoes chain transfer more easily than other radicals existing during the depropagation process. Thus, the terminal alkyl radical is supposed to abstract hydrogen from polymer chain and regenerate polymer on-chain radical and successive $\beta$-scission.
\end{abstract}

KEY WORDS Poly(methyl methacrylate) / Degradation / Photoinitiation / Benzophenone / First-Order Termination /

Poly(methyl methacrylate), PMMA, is a typical polymer which depolymerizes producing mainly monomer in the temperature range of thermal degradation. It has been shown that there are two types of initiation reactions of PMMA in vacuum: $:^{1-3}$ one is chain-end initiated reaction due to double bond at polymer chain ends introduced during the disproportionation termination step of free radical polymerization of methyl methacrylate. The other is a main-chain scission at random position of polymer chains at temperature exceeding $270^{\circ} \mathrm{C}$. Thus, PMMA prepared with anionic polymerization showed by thermogravimetry much higher thermal stability than PMMA polymerized with free radical mecha- nism. ${ }^{3}$ The head-to-head structure probably present in a small amount was also suggested as a site of initiation. ${ }^{4}$ After the initiation reaction, depropagation and termination follow. In contrast to the study on polystyrene degradation, most previous studies on the thermal degradation of PMMA were based only on the weight loss measurement ${ }^{5-7}$ except a few cases. ${ }^{8}$ Recently Kashiwagi et al..${ }^{9}$ gave the activation energy for random scission initiation $\left(233 \mathrm{~kJ} \mathrm{~mol}^{-1}\right)$ and the average zip length $(2620-1340)$ for the thermal degradation of radically polymerized PMMA at $200-325^{\circ} \mathrm{C}$ by weight loss and gel permeation chromatography (GPC) measurements. The initiation step of radically-polymerized PMMA

* To whom all correspondence should be addressed. 
is composed of both chain-end initiation and random main-chain scission. But irrespective of the mode of chain scission, the initiation step has the highest activation energy among elementary reactions of PMMA degradation. All subsequent elementary reactions such as depropagation, termination, chain-transfer, and $\beta$-scission have lower activation energies, and therefore it has been difficult for a long time to analyze clearly these elementary reactions at the thermal degradation temperatures.

The present series of work is based on the idea that if we replace this thermal initiation by photochemical one, we can control the reaction temperature easily. In other words, by the control of the rate of photoinitiation and by carrying out the decomposition in the lower temperature range, we can observe rather easily elementary reactions other than initiation. In the previous paper, ${ }^{10}$ polystyrene was decomposed at $160-270^{\circ} \mathrm{C}$ by photoinitiation method, and relative kinetic rate constants as well as activation energies were determined for all the elementary steps.

The present paper is concerned with the photoinitiated thermal degradation of anionically-prepared PMMA. By this method degradation takes place at temperatures as low as at $140-180^{\circ} \mathrm{C}$. Kinetic parameters and the mechanism of degradation of PMMA are obtained and discussed on the basis of measurements of the decrease in the amount of photoinitiator, change in the molecular weight of PMMA, and weight loss of the sample during degradations.

\section{EXPERIMENTAL}

\section{Materials}

Standard poly(methyl methacrylate), PMMA, with narrow molecular weight distribution $\left(M_{n 0}=8.4 \times 10^{5}, 2.4 \times 10^{5}, 1.3 \times 10^{5}\right.$, $\left.6.6 \times 10^{4} ; M_{w 0} / M_{n 0} \cong 1.1\right)$ and with no terminal double bond prepared by anionic polymerization was purchased from General Science
Coop. Bis(4-benzoylphenyl)ether as an photoinitiator was kindly supplied from Adeka Argus Chemical Co. PMMA films with $5 \mu \mathrm{m}$ thickness containing 0.19 base-mol $1^{-1}$ of the photoinitiator were solvent cast onto glass plate from dichloromethane solution.

\section{Photoinitiated Thermal Degradation}

The film sample set in the reaction vessel under the nitrogen atmosphere was irradiated by $365 \mathrm{~nm}$ light from a 250-W ultrahigh pressure mercury lamp (Ushio USH-250D) with glass filters (Toshiba UV29 and UVD36A). The reaction vessel was heated up to $140-180^{\circ} \mathrm{C}$ with a Supersalt M-2 type salt bath regulated by a thyristor regulator (Chino DF163). Actinometry was carried out with a photometer (Ushio UIT-101) calibrated by the potassium ferrioxalate/o-phenanthroline system. The incident light intensity was $1.6 \times 10^{-8}$ Einstein $\mathrm{cm}^{-2} \mathrm{~s}^{-1}$ in most of the present experiments.

\section{Detection of the Progress of Degradation}

The changes in molecular weight and molecular weight distribution of PMMA after photoirradiation were measured by a TSK HLC-802UR type gel permeation chromatograph (GPC) with a GM1XH6 column and a UV $(254 \mathrm{~nm})$ detector in tetrahydrofuran at $40^{\circ} \mathrm{C}$. The calibration for GPC elution volume was carried out by using standard narrow-molecular-weight-distribution PMMA with various molecular weights. The weight loss due to monomer formation during the degradation of the sample film was measured with a Sartorius microbalance. The change in the amount of aromatic ketone photoinitiator in the sample film was measured by a Shimadzu MPS-5000 type spectrophotometer.

\section{RESULTS AND DISCUSSION}

\section{Photoinitiation Step of Degradation}

Typical change in the absorption spectra of the photosensitizer, $\mathrm{S}$, during the photo- 
initiated thermal degradation of PMMA is shown in Figure 1 together with the absorption spectrum of PMMA film without the photoinitiator. The first-order plots for the change in the aromatic ketone concentration estimated from the decrease in $\pi-\pi^{*}$ absorbance at $290 \mathrm{~nm} \quad\left(\varepsilon_{\mathrm{ab}}=1.4 \times 10^{4} 1 \mathrm{~mol}^{-1} \mathrm{~cm}^{-1}\right)$ gave straight lines at each experimental temperature (Figure 2). The same slopes were obtained for PMMA with different molecular weights measured at $160^{\circ} \mathrm{C}$.

The primary processes for the decay of the aromatic ketone triplet, ${ }^{3} S^{*}$, in the present system are given by eq $1-4$,

$S \stackrel{h v}{\longrightarrow}{ }^{1} S^{*} \longrightarrow{ }^{3} S^{*}$

${ }^{3} S * \stackrel{k_{0}}{\longrightarrow} S$

deactivation

${ }^{3} S^{*}+P \stackrel{k_{\mathrm{q}}}{\longrightarrow} S+P \quad$ physical quenching

${ }^{3} S^{*}+P \stackrel{k_{\mathrm{a}}}{\longrightarrow} \dot{S}+\dot{P}_{\mathrm{c}}$ hydrogen abstraction $(4)$

where $P$ is PMMA, $\dot{S}$ is ketyl radical of $S$, and $\dot{P}_{\mathrm{c}}$ is the on-chain radical of PMMA. The physical quenching of benzophenone triplet by side-chain ester group of PMMA is a predominant process of the deactivation of triplet benzophenone at ambient temperatures, ${ }^{11}$ but hydrogen abstraction of benzophenone triplet probably from methylene group or $\alpha$-methyl group of PMMA becomes evident at elevated temperatures. ${ }^{12}$ The rate of disappearance of aromatic ketone concentration, $[\mathrm{S}]$, under the photostationary state is expressed by

$$
-\mathrm{d}[\mathrm{S}] / \mathrm{d} t=k_{\mathrm{a}}[\mathrm{P}]\left[{ }^{3} S^{*}\right]=I_{\mathrm{ab}} \Phi_{\mathrm{a}}
$$

where intensity of absorbed light, $I_{\mathrm{ab}}$, is given by eq 6 ,

$$
I_{\mathrm{ab}}=10^{3} \sum_{\lambda}\left\{I_{0}\left(1-10^{-\varepsilon_{\mathrm{ab}}[\mathrm{S}] l}\right) / l\right\}
$$

$\Phi_{\mathrm{a}}$ is the apparent quantum yield of ketone disappearance, $\varepsilon_{\mathrm{ab}}$ is the molar extinction

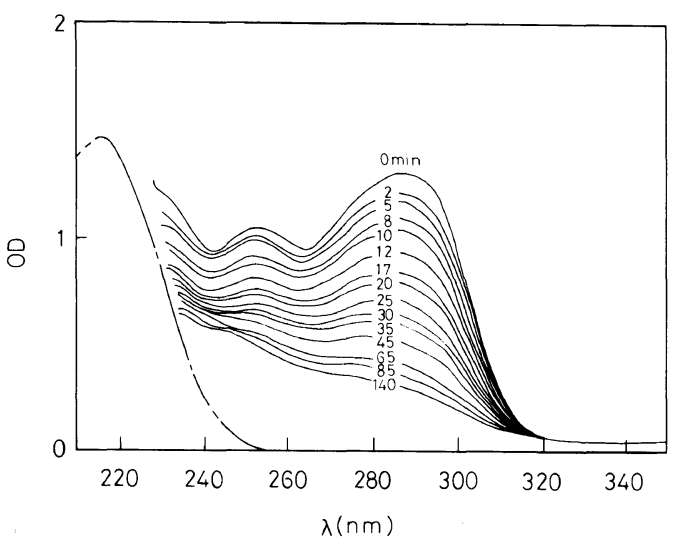

Figure 1. Change in UV spectra of bis(4-benzoylphenyl)ether as a photoinitiator on PMMA film during $365 \mathrm{~nm}$ light irradiation at $160^{\circ} \mathrm{C} . M_{n 0}=8.4 \times 10^{5}$. Irradiation times are shown in the Figure. The dotted line corresponds to absorption of pure PMMA film.

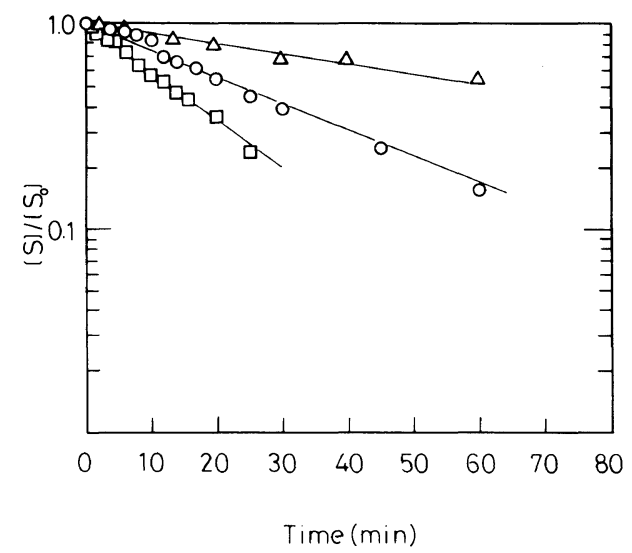

Figure 2. First-order plots of change in aromatic ketone concentration, [S], in PMMA during photoirradiation at $140^{\circ} \mathrm{C}(\triangle), 160^{\circ} \mathrm{C}(\bigcirc)$, and $180^{\circ} \mathrm{C}(\square) . M_{n 0}=8.4 \times 10^{5}$, $I_{0}=1.6 \times 10^{-8}$ Einstein $\mathrm{cm}^{-2} \mathrm{~s}^{-1}$.

coefficient, $I_{0}$ is the intensity of incident light in Einstein $\mathrm{cm}^{-2} \mathrm{~s}^{-1}$, and $l$ is the film thickness in $\mathrm{cm}$. For the case of sufficient transmission of incident light through the film $\left(\varepsilon_{\mathrm{ab}}[\mathrm{S}] l<0.1\right)$ which holds in the present experiments, eq 5 and 6 result in eq 7 ,

$$
\ln \left([\mathrm{S}] /[\mathrm{S}]_{0}\right)=-2.3 \times 10^{3} \Phi_{\mathrm{a}} t \sum_{\lambda}\left(I_{0} \varepsilon_{\mathrm{ab}}\right)=-\alpha t
$$

and $\Phi_{\mathrm{a}}$ can be obtained from the slope, $\alpha$, in 
Table I. Apparent quantum yield for initiator consumption, $\Phi_{\mathrm{a}}$, number of depolymerized monomer units per ketone group consumed, $\Delta M / \Delta S$, number of main-chain scissions per ketone group consumed, $Z_{\mathrm{s}} M_{0} / \Delta S$, and zip length, $\Delta W / Z_{\mathbf{s}}$, as well as their activation energies for photoinitiated thermal degradation of poly(methyl methacrylate) with $M_{n 0}=8.4 \times 10^{5}$

\begin{tabular}{|c|c|c|c|c|c|}
\hline \multicolumn{2}{|c|}{$\begin{array}{c}I_{0} \times 10^{8} \\
(\text { Einstein/ } \\
\left.\mathrm{cm}^{2} \mathrm{~s}\right)\end{array}$} & $\Phi_{\mathrm{a}}$ & $\Delta M / \Delta S$ & $Z_{\mathbf{s}} M_{0} / \Delta S$ & $\Delta W / Z_{\mathrm{s}}$ \\
\hline $140^{\circ} \mathrm{C}$ & 1.6 & $1.9 \times 10^{-3}$ & 23.2 & 0.029 & $0.79 \times 10^{3}$ \\
\hline \multirow[t]{4}{*}{$160^{\circ} \mathrm{C}$} & 0.71 & $5.1 \times 10^{-3}$ & & & \\
\hline & 1.6 & $4.5 \times 10^{-3}$ & 31.3 & 0.022 & $1.42 \times 10^{3}$ \\
\hline & 2.7 & $2.8 \times 10^{-3}$ & & & \\
\hline & 6.6 & $2.2 \times 10^{-3}$ & & & \\
\hline $180^{\circ} \mathrm{C}$ & 1.6 & $8.8 \times 10^{-3}$ & 62.6 & 0.020 & $3.19 \times 10^{3}$ \\
\hline \multicolumn{2}{|c|}{$\Delta E\left(\mathrm{kcal} \mathrm{mol}^{-1}\right)$} & 13.5 & 8.7 & -3.4 & 12.3 \\
\hline
\end{tabular}

Figure 2. The summation in $\sum_{\lambda}\left(I_{0} \varepsilon_{\mathrm{ab}}\right)$ is done with the wave lengths, $\lambda$, of the incident light.

The value of $\Phi_{\mathrm{a}}$ for hydrogen abstraction from PMMA are given in Table $I$ with the activation energy of $13.5 \mathrm{kcal} \mathrm{mol}^{-1}$. It should be noted that $\Phi_{\mathrm{a}}$ decreases with increasing incident light intensity, $I_{0}$. The intensity exponent, $\eta$, in the $I_{0}$ dependence of the rate of initiator disappearance $(\mathrm{d}[\mathrm{S}] /$ $\left.\mathrm{d} t \propto I_{0}{ }^{\eta}\right)$ seems to be 0.6 at $160^{\circ} \mathrm{C}$, which agrees with the previous observation of $\eta=0.52$ for benzophenone $^{11}$ in PMMA at $20^{\circ} \mathrm{C}$. The stationary-state irradiation by high-pressure mercury lamp in these cases would have produced a high concentration of triplet benzophenone leading to its deactivation through triplet-triplet annihilation. This would explain the smaller intensity exponent than unity for benzophenone disappearance in PMMA.

\section{Depolymerization and Main-Chain Scission}

The weight loss, $\Delta W$, of PMMA films ( $5 \mu \mathrm{m}$ thickness) containing 0.19 base-moll ${ }^{-1}$ of photoinitiator during the photoirradiation at $140-180^{\circ} \mathrm{C}$ is shown in Figure 3 for a polymer

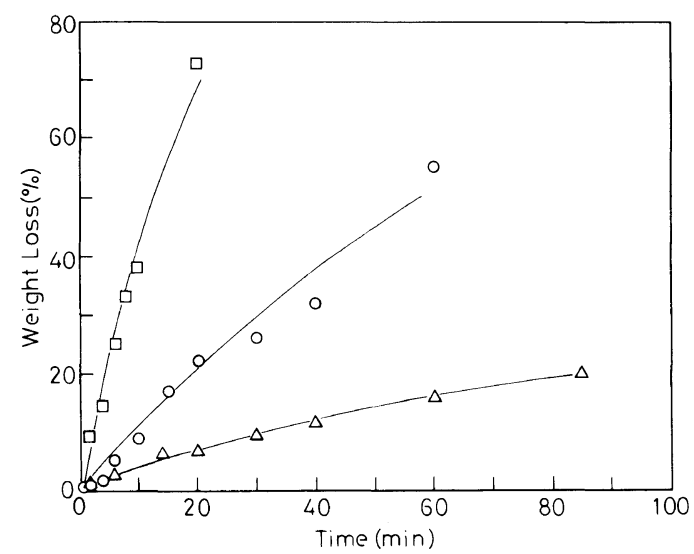

Figure 3. Weight loss of PMMA film during photoinitiated thermal degradation at $140^{\circ} \mathrm{C}(\triangle), 160^{\circ} \mathrm{C}(\bigcirc)$, and $180^{\circ} \mathrm{C}(\square) . M_{n 0}=8.4 \times 10^{5}, S_{0}=0.19$ base-moll $^{-1}$.

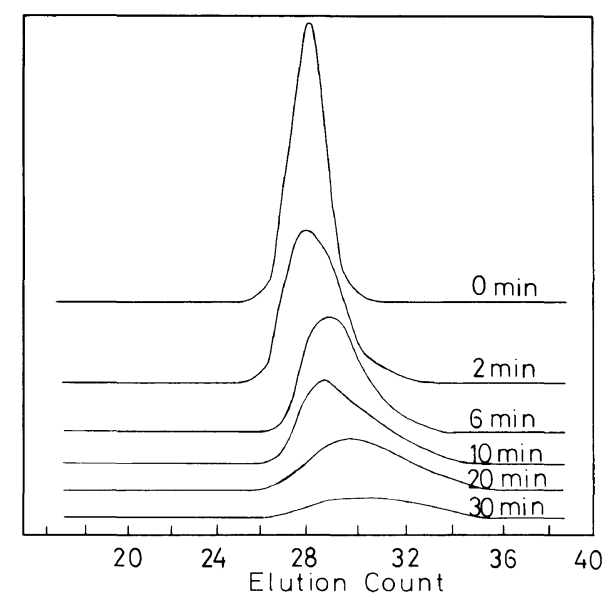

Figure 4. Change in GPC curves of PMMA after photoinitiated thermal degradation at $160^{\circ} \mathrm{C}$ for the times indicated beside the curves. $M_{n 0}=8.4 \times 10^{5}$.

with $M_{n 0}=8.4 \times 10^{5}$. The depolymerization usually observed at temperatures higher than $300^{\circ} \mathrm{C}$ takes place at $140-180^{\circ} \mathrm{C}$ in the present case of photoinitiated degradation and exhibits marked temperature dependence. More than $70 \%$ of the monomer units were vaporized at $180^{\circ} \mathrm{C}$ under an atomic pressure of nitrogen by 20-min photoirradiation.

Typical change in GPC curves for photoirradiated PMMA is given in Figure 4 and the changes in number-average, $\bar{M}_{n}$, and weight- 


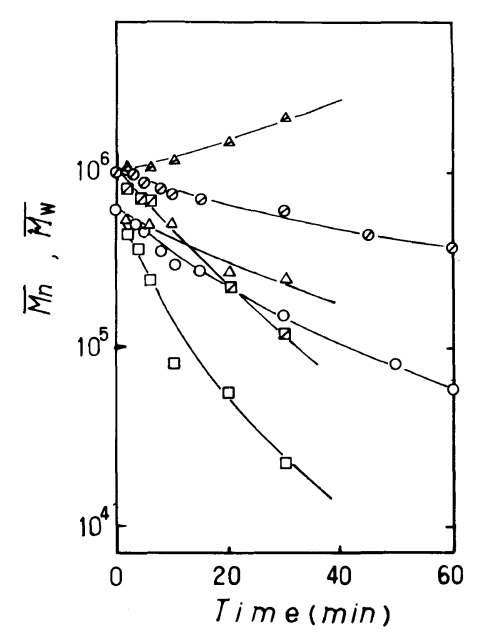

Figure 5. Changes in the number-average moleculer weight, $\bar{M}_{n},(\triangle, \bigcirc, \square)$ and weight-average molecular weight, $\bar{M}_{w},(\triangle, \oslash, \nabla)$ of PMMA during photoinitiated thermal degradation at $140^{\circ} \mathrm{C}(\triangle, \triangle), 160^{\circ} \mathrm{C}(O, \oslash)$, and $180^{\circ} \mathrm{C}(\square, \square) . M_{n 0}=8.4 \times 10^{5}$.

average, $\bar{M}_{w}$, molecular weights are illustrated in Figure 5. In the present work, $M_{n}$ decreases during the photoinitiated degradations, since the zip length of depolymerization is not sufficient for complete unzipping. The depolymerization proceeds after the $\beta$-scission of on-chain radicals produced by the hydrogen abstraction. The number of main-chain scissions per monomer unit is usually given by

$$
Z_{\mathrm{s}}^{\prime}=1 / N-1 / N_{0}
$$

where $N$ is the number-average degree of polymerization and $N_{0}$ is its initial value. Equation 8 is valid for a case where there is no occurrence of weight loss due to depolymerization, but when depolymerization proceeds during the degradation, the effect of weight loss on the number of main-chain scissions should be considered.

The total moles of the main chain scission in the system, $Z$, and number of main chain scissions per monomer unit, $Z_{\mathrm{s}}$, are given by eq 9 and 10 for a case in which $N_{0} / 2$ is larger than the kinetic chain length, $v$,

$$
Z=P-P_{0}=M / N-M_{0} / N_{0} \quad\left(\text { for } N_{0} / 2 \geqq v\right)(9)
$$

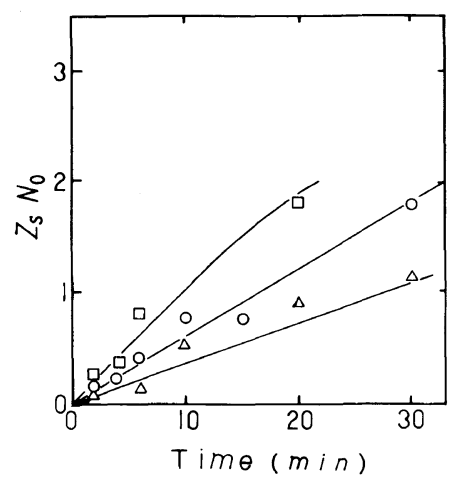

Figure 6. Change in the number of main-chain scissions per polymer chain, $Z_{\mathrm{s}} N_{0}$, during photoinitiated thermal degradation of PMMA at $140^{\circ} \mathrm{C}(\triangle), 160^{\circ} \mathrm{C}(\bigcirc)$, and $180^{\circ} \mathrm{C}(\square) . M_{n 0}=8.4 \times 10^{5}, S_{0}=0.19$ base-moll ${ }^{-1} I_{0}=$ $1.6 \times 10^{-8}$ Einstein $\mathrm{cm}^{-2} \mathrm{~s}^{-1}$.

$$
Z_{\mathrm{s}}=Z / M_{0}=(1 / N)\left(M / M_{0}\right)-1 / N_{0}
$$

where $P$ and $M$ are moles of polymer and base mole of the repeating unit in the system, respectively, $P_{0}$ and $M_{0}$ are their initial values. The kinetic chain length, $v$, is defined as the number of monomer units depolymerized per on-chain radical initially produced by the hydrogen abstraction by benzophenone triplet. As the fractional weight loss, $\Delta W$, is expressed in eq 11 ,

$$
\Delta W=1-M / M_{0}
$$

we get $Z_{\mathrm{s}}$ as eq 12 .

$$
\begin{aligned}
Z_{\mathrm{s}} & =\{(1-\Delta W) / N\}-1 / N_{\mathrm{o}} \\
& =(1-\Delta W) Z_{\mathrm{s}}^{\prime}-\Delta W / N_{0}
\end{aligned}
$$

For the case of complete depolymerization $\left(N_{0} / 2 \leqq v\right), Z$ and $Z_{\mathrm{s}}$ are expressed in eq 13 and 14.

$$
\begin{aligned}
& Z=P-P_{0}+2\left(M_{0}-M\right) / N_{0}\left(\text { for } N_{0} / 2 \leqq v\right) \\
& Z_{\mathrm{s}}=(1-\Delta W) Z_{\mathrm{s}}^{\prime}+\Delta W / N_{0}
\end{aligned}
$$

Thus, the value of $Z_{\mathrm{s}}$ including the effect of weight loss can be calculated from the results of GPC and weight loss measurements. The time-course of the number of main-chain scission per initial polymer chain, $Z_{\mathrm{s}} N_{0}$, is shown in Figure 6. The rate of main-chain 
scission increases with temperature in contrast to the case of thermal degradation of polystyrene, ${ }^{10}$ where the large activation energy for bimolecular termination step of degradation results in a negative activation energy for the overall rate of main-chain scission. The zip length of depolymerization, $\Delta W / Z_{\mathrm{s}}$, i.e., number of depolymerized monomers units per on-chain radical formed by both hydrogen abstraction of bebzophenone triplet and chain transfer from terminal radical can be calculated from eq 15 for the case of $N_{0} / 2 \geqq$ $v$, where the kinetic chain length, $v$, is equal to zip length, $\Delta W / Z_{\mathrm{s}}$.

$$
\begin{gathered}
\Delta W / Z_{\mathrm{s}}=v=\Delta W /\left\{(1-W) / N-1 / N_{0}\right\} \\
\left(\text { for } N_{0} / 2 \geqq v\right)
\end{gathered}
$$

Some kinetic parameters such as a number of depolymerized monomer units per ketone group consumed, $\Delta M / \Delta S$, number of mainchain scissions per ketone group consumed, $Z_{\mathrm{s}} M_{0} / \Delta S$, and zip length, $\Delta W / Z_{\mathrm{s}}$, are summarized in Table $I$ together with their activation energies. The zip length, $\Delta W / Z_{\mathrm{s}}$, increases with temperature and lies in the range of $(0.8-$ $3.2) \times 10^{3}$ for $140-160^{\circ} \mathrm{C}$ for PMMA with $M_{n 0}=8.4 \times 10^{5}$. The number of main-chain scissions per ketone groups consumed, $Z_{\mathrm{s}} M_{0} /$ $\Delta S$, is very small, of the order of $10^{-2}$ and shows a small negative temperature dependence. The values of $\Delta M / \Delta S, Z_{\mathrm{s}} M_{0} / \Delta S$, $\Delta W / Z_{\mathrm{s}}$ are independent of incident light intensity, $I_{0}$, as can be seen from in Figures 10 and 11 .

\section{Mechanism of Photoinitiated Thermal Deg- radation of $P M M A$}

The possible reactions of PMMA on-chain radical, $\dot{P}_{c}$, including its formation by hydrogen abstraction and escape from cage are summarized as follows:

$$
\begin{aligned}
& { }^{3} S^{*}+P \stackrel{k_{\mathrm{a}}{ }^{\longrightarrow}}{\longrightarrow}\left(\dot{S}^{\dot{S}} \dot{P}_{\mathrm{c}}\right)_{\mathrm{cage}} \stackrel{f}{\longrightarrow} \dot{S}+\dot{P}_{\mathrm{c}} \\
& \dot{P}_{\mathrm{c}} \stackrel{k_{\beta}}{\longrightarrow} \dot{P}_{\mathrm{e}}^{i}+P \quad \beta \text {-scission }
\end{aligned}
$$

$\dot{P}_{\mathrm{e}}^{i} \stackrel{k_{\mathrm{d}}}{\longrightarrow} \dot{P}_{\mathrm{e}}^{i-1}+M$ depropagation

$\dot{P}_{\mathrm{e}}^{1} \stackrel{k_{\mathrm{d}}}{\longrightarrow} \dot{R}+M$

$\dot{P}_{\mathrm{e}}+P \stackrel{k_{\mathrm{f}}}{\longrightarrow} P+\dot{P}_{\mathrm{c}} \quad$ chain-transfer

$\dot{R}+P \stackrel{k_{\mathrm{f}}^{\prime}}{\longrightarrow} \dot{P}_{\mathrm{c}}+R H \quad$ chain transfer from terminal radical

where $\dot{P}$ is the PMMA radical covering both on-chain $\left(\dot{P}_{\mathrm{c}}\right)$ and end $\left(\dot{P}_{\mathrm{e}}\right)$ radicals $\left(\dot{P}=\dot{P}_{\mathrm{c}}+\right.$ $\left.\dot{P}_{\mathrm{e}}\right), \dot{S}$ is ketyl radical of photoinitiator and $f$ is the intiator efficiency. The polymer on-chain radical undergoes a $\beta$ scission to produce the chain end radical, which mainly depolymerizes, but some of the on-chain radical also undergo chain transfer reproducing the on-chain radical or terminate to unreactive products. Since the temperature range in the present study is below or nearly equal to the ceiling temperature of PMMA, the polymerization process might be partly included in the depropagation step (eq 18) as a backward reaction, though monomer concentration in the sample is low due to vaporization. In the present experiments, the on-chain radical would be mainly (A) or (B) or both, providing the depropagating end radical (C). If hydrogen is transferred from the ester methyl group, the radical (D) results in on-chain radical (E) and hence tertiary end radical $(\mathrm{F})$.<smiles>[CH]C(CC(C)(C)C(=O)OC)C(=O)OC</smiles>
(A)
(B)
(C) 
<smiles>CCC(C)(C)C(=O)OC</smiles>

(D)<smiles>COC(=O)C(C)CCC(C)C</smiles>

(E)
Equations 19 and 21 correspond to the formation and chain transfer of the terminal radical unable to depolymerize, and the rate coefficient of chain transfer $k_{\mathrm{f}}^{\prime}$ of the terminal radical, $\dot{R}$, can be different from $k_{\mathrm{f}}$. The termination step is usually considered to be second-order reactions between radical pairs. If the termination reactivity of $\dot{R}$ is the same as that of $S$, the termination step is given by eq 22 , showing the second-order termination mechanism.

$$
\left.\begin{array}{l}
\dot{P}+\dot{P} \stackrel{k_{\mathrm{t} 1}}{\longrightarrow} \\
\dot{P}+\dot{S} \stackrel{k_{\mathrm{t} 2}}{\longrightarrow} \\
\dot{S}+\dot{S} \stackrel{k_{\mathrm{t} 3}}{\longrightarrow}
\end{array}\right\} \text { termination }
$$

In the thermal degradation of PMMA, however, Jellinek ${ }^{7}$ and Kashiwagi et al. ${ }^{9}$ suggested termination of a polymer radical with a monomer, leading to a first-order termination mechanism (eq 23). This will be discussed later in detail.

$$
\dot{P} \stackrel{k_{\mathrm{t}}}{\longrightarrow} P
$$

Now we examine the kinetics based on each termination mechanism. When we assume the first-order termination, the rate of initiation, $R_{i}$, is given by eq 24 by using steady-state assumption for polymer radical,

$$
\begin{aligned}
R_{i} & =f k_{\mathrm{a}}\left[{ }^{3} S^{*}\right] / v_{0} \\
& =f \Phi \times 2.3 \times 10^{3}\left(\sum_{\lambda} I_{0} \varepsilon_{\mathrm{ab}}\right) S=f \alpha S=k_{\mathrm{t}} \dot{P}
\end{aligned}
$$

where $v_{0}$ is molar volume of repeating unit, $S$ is the amount of photoinitiator, and $\dot{P}$ is the stationary-state amount of polymer radical. The amount of chain-end radical, $\dot{P}_{\mathrm{e}}$, is then given by

$$
\dot{P}_{\mathrm{e}}=r_{\mathrm{e}} \dot{P}=\left[k_{\beta} /\left(k_{\mathrm{f}} / v_{0}+k_{\beta}+k_{\mathrm{t}}\right)\right] \dot{P}
$$

where $r_{\mathrm{e}}$ is the fraction of end radicals among whole polymer radicals. For the case of $k_{\beta} \gg$ $k_{\mathrm{t}} \gg k_{\mathrm{f}} / v_{0}$, eq 26 holds

$$
\dot{P}_{\mathrm{e}} \cong \dot{P}=R_{i} / k_{\mathrm{t}}, \quad \dot{P}_{\mathrm{c}} \cong\left(k_{\mathrm{t}} / k_{\beta}\right) \dot{P}=R_{i} / k_{\beta}
$$

The rate of the main-chain scission per repeating unit, $\mathrm{d} Z_{\mathrm{s}} / \mathrm{d} t$, and the rate of weight loss, $-\mathrm{d} V / \mathrm{d} t$, are given by eq $27-28$, respectively.

$$
\begin{gathered}
\mathrm{d} Z_{\mathrm{s}} / \mathrm{d} t=k_{\beta} \dot{P}_{\mathrm{c}} / M_{0}=R_{i} v_{0} / V_{0}=f \alpha S v_{0} / V_{0} \\
=-\left(f v_{0} / V_{0}\right)(\mathrm{d} S / \mathrm{d} t) \\
\begin{aligned}
(\mathrm{d} V / \mathrm{d} t)=k_{\mathrm{d}} \dot{P}_{\mathrm{e}} v_{0}=k_{\mathrm{d}}\left(R_{i} / k_{\mathrm{t}}\right) v_{0} \\
=\left(k_{\mathrm{d}} V_{0} / k_{\mathrm{t}}\right)\left(\mathrm{d} Z_{\mathrm{s}} / \mathrm{d} t\right)
\end{aligned}
\end{gathered}
$$

where $V$ is the volume of the system and $V_{0}$ is its initial value. The integration of eq 27 and 28 leads to

$$
\begin{gathered}
Z_{\mathrm{s}}=\left(f v_{0} / V_{0}\right)\left(S_{0}-S\right) \\
\left(V_{0}-V\right) / V_{0}=\left(k_{\mathrm{d}} / k_{\mathrm{t}}\right) Z_{\mathrm{s}}
\end{gathered}
$$

For the case of $k_{\beta} \gg k_{f} / v_{0}>k_{\mathrm{t}}, \dot{P}_{\mathrm{e}}$ and $\dot{P}_{\mathrm{c}}$ are given by

$$
\dot{P}_{\mathrm{e}}=R_{i} / k_{\mathrm{t}}, \quad \dot{P}_{\mathrm{c}}=\left(R_{i} / k_{\mathrm{t}}\right)\left(k_{\mathrm{f}} / k_{\beta} v_{0}\right)
$$

and eq 32 and 33 can be derived in place of eq 29 and 30.

$$
Z_{\mathrm{s}}=\left(f k_{\mathrm{f}} / k_{\mathrm{t}} V_{0}\right)\left(S_{0}-S\right)
$$

$\left(V_{0}-V\right) / V_{0}=\left(k_{\mathrm{d}} v_{0} / k_{\mathrm{f}}\right) Z_{\mathrm{s}}$

Equation 27 suggests that the temperature dependence of $\mathrm{d} Z_{\mathrm{s}} / \mathrm{d} t$ is governed by that of the initiation step, $f \alpha$, shown in Figure 6. Linear relationship for $Z_{\mathrm{s}} v s$. $\left(S_{0}-S\right)$ and $\left(V_{0}-V\right)$ vs. $Z_{\mathrm{s}}$ are suggested according to eq 29 and 30 or eq 32 and 33 with slopes independent of irradiation intensity.

When we assume the second-order termination, the rate of initiation, $R_{i}$, and the sta- 
tionary state amount of polymer radical, $\dot{P}$, are given by

$$
\begin{aligned}
R_{i} & \left.=f k_{\mathrm{a}}{ }^{3} S^{*}\right] / v_{0}=f \phi \times 2.3 \times 10^{3}\left(\sum_{\lambda} I_{0} \varepsilon_{\mathrm{ab}}\right) S \\
& =f \alpha S=k_{\mathrm{T}} \dot{P}^{2} / V \\
\dot{P} & =\left\{k_{\mathrm{t} 1}+k_{\mathrm{t} 2}\left(k_{\mathrm{t} 1} / k_{\mathrm{t} 3}\right)^{1 / 2}\right\}^{-1 / 2}(V f \alpha S)^{1 / 2} \\
& =\left(V f \alpha S / k_{\mathrm{T}}\right)^{1 / 2}
\end{aligned}
$$

where $k_{\mathrm{T}}=k_{\mathrm{t} 1}+k_{\mathrm{t} 2}\left(k_{\mathrm{t} 1} / k_{\mathrm{t} 3}\right)^{1 / 2}$ is the reduced termination rate coefficient. The amount of chain end radical, $\dot{P}_{\mathrm{e}}$, is then expressed in eq 36 .

$$
\dot{P}_{\mathrm{e}}=r_{\mathrm{e}} \dot{P}=\left\{k_{\beta} /\left[k_{\mathrm{f}} / v_{0}+k_{\beta}+\left(R_{i} k_{\mathrm{T}} / V\right)^{1 / 2}\right]\right\} \dot{P} \text { (36) }
$$

For the case of $k_{\beta} \gg\left(R_{i} k_{\mathrm{T}} / V\right)^{1 / 2}>k_{\mathrm{f}} / v_{0}$, the relationships among $Z_{\mathrm{s}}, S$, and $V$ are given by eq 29,37 , and 38 ,

$$
\begin{aligned}
& V_{0}^{1 / 2}-V^{1 / 2}=v_{0} k_{\mathrm{d}}\left(f / \alpha k_{\mathrm{T}}\right)^{1 / 2}\left(S_{0}{ }^{1 / 2}-S^{1 / 2}\right) \\
& \left(V_{0}-V\right) / V_{0} \\
& \quad=k_{\mathrm{d}}\left(f \alpha S_{0} k_{\mathrm{T}}\right)^{-1 / 2} Z_{\mathrm{s}}\left(1-A \cdot Z_{\mathrm{s}}+\cdots\right)
\end{aligned}
$$

For the case of $k_{\beta} \gg k_{\mathrm{f}} / v_{0}>\left(R_{i} k_{\mathrm{T}} / V\right)^{1 / 2}$, eq 33 holds, and the relationship between $Z_{\mathrm{s}}$ and $S$ becomes rather complicated as is given by eq 39.

$$
\mathrm{d} Z_{\mathrm{s}} / \mathrm{d} S=k_{\mathrm{f}}\left(f / \alpha S V_{0} k_{\mathrm{T}}\right)^{1 / 2}\left[1-\left(v_{0} k_{\mathrm{d}} / k_{\mathrm{f}}\right) Z_{\mathrm{s}}\right]^{1 / 2}
$$

Thus, the second-order termination mechanism indicates that the relationship between either $V$ and $Z_{\text {s }}$ (eq 38) or $Z_{\text {s }}$ and $S$ (eq 39) depends on the irradiation intensity.

The irradiation intensity dependences of the changes in weight loss and $Z_{\mathrm{s}} N_{0}$ during photoinitiated thermal degradation of PMMA at $160^{\circ} \mathrm{C}$ are shown in Figures 7 and 8 , respectively. The plots of initial slopes in Figures 7 and 8 as well as the rates of initiator disappearance against incident light intensity (Figure 9) shows that intensity exponents for $\mathrm{d} \Delta W / \mathrm{d} t, \mathrm{~d} Z_{\mathrm{s}} / \mathrm{d} t$, and $-\mathrm{d} S / \mathrm{d} t$ are about 0.5 , 0.6 , and 0.6 , respectively. The intensity exponent of $0.45-0.74$ has been reported for the rate of monomer formation in an early work of photodegradation of $\mathrm{PMMA}^{13}$ though a bimolecular termination mechanism

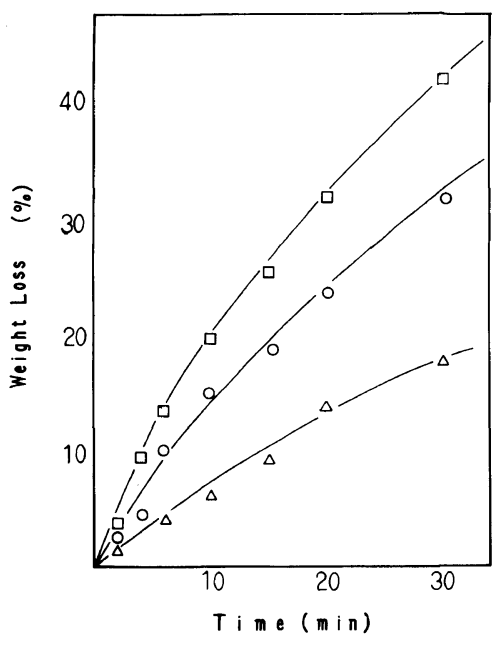

Figure 7. Irradiation intensity dependence of weight loss during photoinitiated thermal degradation of PMMA of $M_{n 0}=2.4 \times 10^{5} . I_{0} / 10^{-8}$ Einstein $\mathrm{cm}^{-2} \mathrm{~s}^{-1}=0.71(\triangle), 2.7$ (○), $6.6(\square)$.

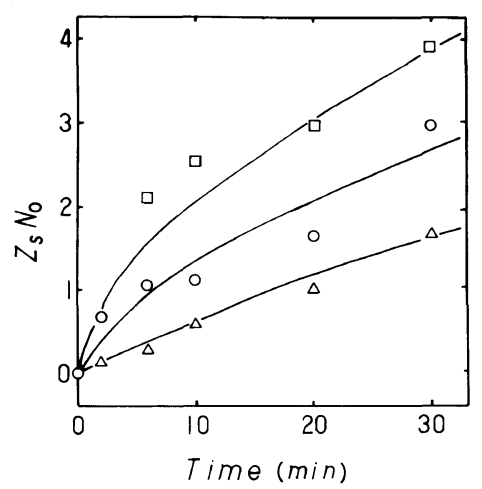

Figure 8. Irradiation intensity dependence of the number of chain scissions per polymer chain, $Z_{\mathbf{s}} N_{0}$, during photoinitiated thermal degradation of PMMA of $M_{n 0}=$ $2.4 \times 10^{5} . I_{0} / 10^{-8}$ Einstein $\mathrm{cm}^{-2} \mathrm{~s}^{-1}=0.71(\triangle), 2.7(\bigcirc)$, $6.6(\square)$.

was suggested from this intensity exponent value in those days. The data in Figures 7 and 8 are replotted in Figures 10 and 11, showing the relationships between $Z_{\mathrm{s}} N_{0}$ vs. $\left(1-S / S_{0}\right)$ and $\left(1-W / W_{0}\right)$ vs. $Z_{\mathrm{s}} N_{0}$, respectively. The linear relationships with no irradiation-intensity dependence in both Figures 10 and 11, therefore, support the first-order termination mechanism given by eq 29 and 30 or eq 32 and 


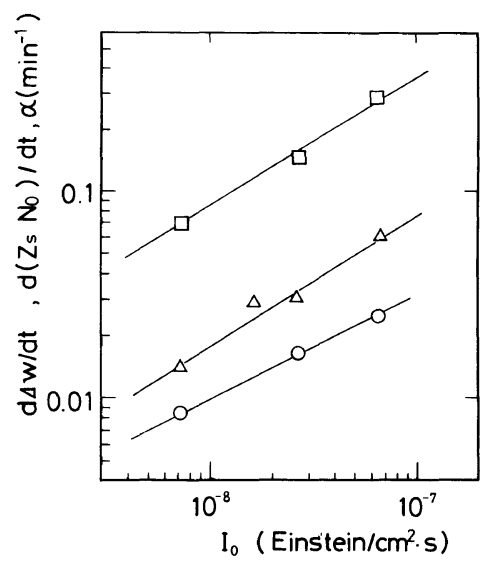

Figure 9. Irradiation intensity dependence of the initial rates of weight less $\mathrm{d} \Delta W / \mathrm{d} t(O)$, main chain scission $\mathrm{d}\left(Z_{\mathrm{s}} N_{0}\right) / \mathrm{d} t(\square)$, and initiator disappearance $\alpha(\triangle)$. $M_{n 0}=2.4 \times 10^{5}, 160^{\circ} \mathrm{C}$.

33. The presence of a large amount of MMA monomer in the system may result in the rapid formation of allyl type stable monomer radical $\left(\Delta E<10 \mathrm{kcal} \mathrm{mol}^{-1}\right)^{3}$ (eq 40 ) compared to the bimolecular terminations between radicals of small concentrations.<smiles>[Y]C=C(C[18O])C(=O)OC</smiles><smiles>[H][R]CC(=C)C(=O)OC</smiles>

The first-order termination was not observed for photo-initiated thermal degradation of polystyrene, ${ }^{10}$ since the formation of allyl type radical is impossible from the styrene monomer. The present results of first-order termination also agree with the same suggestion by Kashiwagi et al.$^{9,14}$ for thermal degradation of PMMA at $200-360^{\circ} \mathrm{C}$.

The kinetic parameters and their activation energies based on the first-order termination were calculated using the values of tempera-

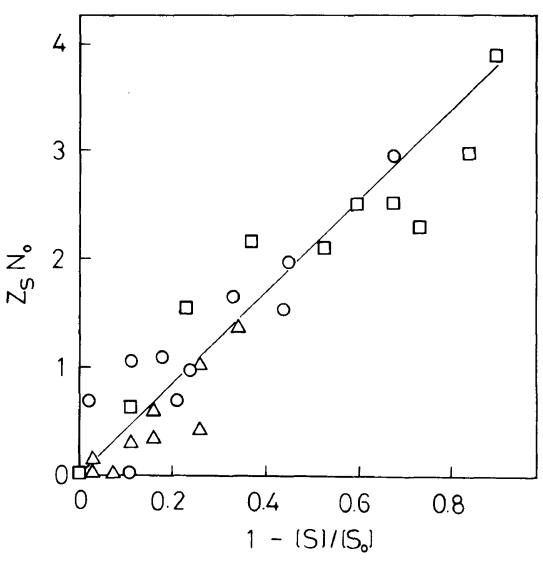

Figure 10. The number of main-chain scissions per polymer chain, $Z_{\mathbf{s}} N_{0}$, plotted against the amount of ketone group consumed, $1-S / S_{0}$, for the degradation of PMMA at $160^{\circ} \mathrm{C}$ with various incident light intensities, $I_{0} . I_{0} / 10^{-8}$ einstein $\mathrm{cm}^{-2} \mathrm{~s}^{-1}=0.71(\triangle), 2.7(\bigcirc), 6.6(\square) . M_{n 0}=$ $2.4 \times 10^{5}$.

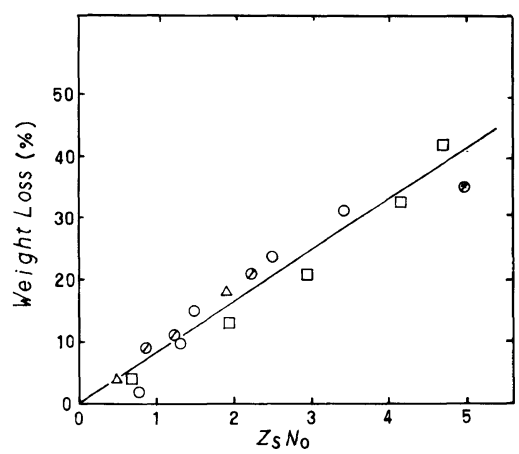

Figure 11. Weight loss, $1-W / W_{0}$, plotted against the number of main-chain scissions per polymer chain, $Z_{\mathrm{s}} N_{0}$, for the degradation of PMMA of $M_{n 0}=2.4 \times 10^{5}$ at $160^{\circ} \mathrm{C}$, with various incident light intensities, $I_{0} . I_{0} / 10^{-8}$ Einstein $\mathrm{cm}^{-2} \mathrm{~s}^{-1}=0.71(\triangle), 1.6(\bigcirc), 2.7(\bigcirc), 6.6(\square)$.

ture dependence in Table I and eq 29 and 30 or eq 32 and 33 together with the values for $k_{\mathrm{d}}{ }^{3}$. The results are summarized in Table II. The value of $\Delta E_{\mathrm{t}} \cong 10-13 \mathrm{kcal} \mathrm{mol}^{-1}$ irrespective of the condition for $k_{\mathrm{t}}$ and $k_{\mathrm{f}} / v_{0}$ is reasonable for the termination of the polymer radical due to hydrogen abstraction from monomer. 
Table II. Kinetic parameters and their activation energies, $\Delta E$, for the photoinitiated thermal degradation of poly(methyl methacrylate)

\begin{tabular}{lllll}
\hline $\begin{array}{c}\text { Tempera- } \\
\text { ture }\end{array}$ & $140^{\circ} \mathrm{C}$ & $160^{\circ} \mathrm{C}$ & $180^{\circ} \mathrm{C}$ & \multicolumn{1}{c}{$\Delta E$} \\
\cline { 4 - 5 } & & & & $\mathrm{kcal} \mathrm{mol}^{-1}$ \\
\hline For $k_{\mathrm{t}}>k_{\mathrm{f}} / v_{0}$ (eq 29 and 30) & & \\
$f$ & 0.029 & 0.022 & 0.020 & -3.4 \\
$k_{\mathrm{d}} / k_{\mathrm{t}}$ & $7.9 \times 10^{2}$ & $1.4 \times 10^{3}$ & $3.2 \times 10^{3}$ & 12.3 \\
$k_{\mathrm{d}}\left(\mathrm{s}^{-1}\right)^{3}$ & $3.0 \times 10^{3}$ & $8.2 \times 10^{3}$ & $2.1 \times 10^{4}$ & 18 \\
$k_{\mathrm{t}}\left(\mathrm{s}^{-1}\right)$ & 3.8 & 5.9 & 6.6 & 9.7 \\
\hline
\end{tabular}

\section{Molecular Weight Dependence of Photoinitiated Thermal Degradation of PMMA}

The initial molecular weight dependence of the photoinitiated thermal degradation of PMMA was investigated in order to gain further insight into the mechanism. In the case of termal degradation of PMMA with the initial degree of polymerization, $N_{0}$, larger than kinetic chain length, $v$, the chain transfer process of terminal radical regenerating an on-chain radical can be neglected, but for PMMA with $N_{0} / 2 \leqq v$ the process of chain transfer of terminal radical becomes important and we must distinguish zip length, $\varepsilon$, from kinetic chain length, $v$. The rate of disappearance of photoinitiator (Figure 12) and the rate of weight loss (Figure 13) during the degradation were observed to be independent of the initial degree of polymerization, $N_{0}$, of PMMA. But the rate of main-chain scission per monomer unit, $Z_{\mathrm{s}}$, increases with decrease in $N_{0}$ (Figure 14), leading to decrease in zip length, $\varepsilon=\Delta W / Z_{\mathrm{s}}$, from 1240 to 105 with decreasing degree of polymerization, $N_{0}$, as is summarized in Table III. The zip length $\varepsilon$ is always less than half $N_{0}$.

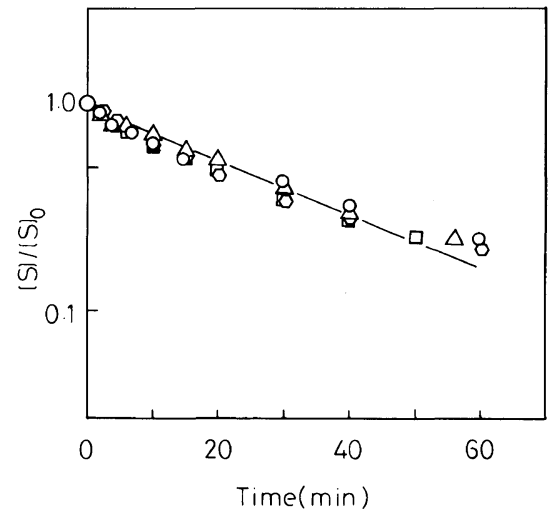

Figure 12. First-order plots of decrease in the aromatic ketone concentration, [S], in PMMA during photoirradiation at $160^{\circ} \mathrm{C} . M_{n 0}=8.4 \times 10^{5}(\bigcirc), 2.4 \times 10^{5}(\triangle)$, $1.27 \times 10^{5}(\bigcirc), 6.6 \times 10^{4}(\square)$.

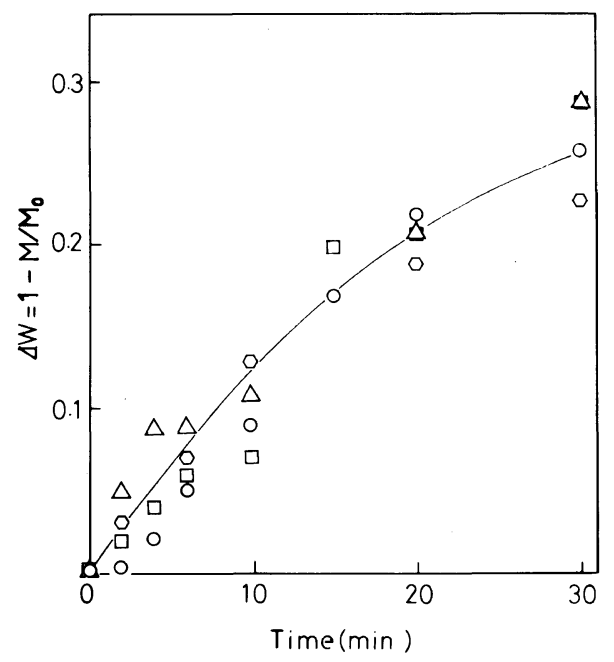

Figure 13. Weight loss of PMMA films with $M_{n 0}=$ $8.4 \times 10^{5}(\mathrm{O}), 2.4 \times 10^{5}(\triangle), 1.3 \times 10^{5}(\mathrm{O})$, and $6.6 \times 10^{4}$ $(\square)$, during photoinitiated thermal degradation at $160^{\circ} \mathrm{C}$. $I_{0}=1.7 \times 10^{-8}$ Einstein $\mathrm{cm}^{-2} \mathrm{~s}^{-1}, S_{0}=0.19$ base-moll ${ }^{-1}$.

Three cases for the behavior of terminal radical, $\dot{R}$, are now examined on the basis of first-order termination for explanation of these phenomena. Case (A) corresponds to the equal reactivity of $\dot{R}$ to that of $\dot{P}_{\mathrm{e}}^{i}\left(k_{\mathrm{f}}^{\prime}=k_{\mathrm{f}}, k_{\mathrm{t}}^{\prime}=k_{\mathrm{t}}\right)$, where $k_{\mathrm{f}}^{\prime}$ and $k_{\mathrm{t}}^{\prime}$ are the rate coefficients for eq 21 and 41 , respectively.

$$
\dot{R} \stackrel{k_{\mathrm{t}}^{\prime}}{\longrightarrow} R
$$




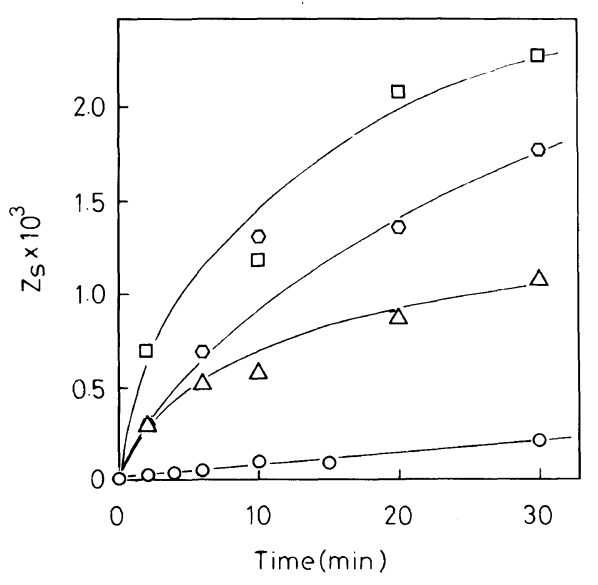

Figure 14. Change in the number of main-chain scissions per monomer unit, $Z_{\mathrm{s}}$, during photoinitiated thermal degradation of PMMA at $160^{\circ} \mathrm{C}$ with $M_{n 0}=8.4 \times 10^{5}(\mathrm{O})$, $2.4 \times 10^{5}(\triangle), 1.3 \times 10^{5}(\bigcirc), 6.6 \times 10^{4}(\square) . \quad S_{0}=0.19$ base-moll $1^{-1}, I_{0}=1.7 \times 10^{-8}$ Einstein $\mathrm{cm}^{-2} \mathrm{~s}^{-1}$.

In case (B) chain transfer from $\dot{R}$ to polymer chain (eq 21) is much faster than that from $\dot{P}_{\mathrm{e}}$ $\left(k_{\mathrm{f}}^{\prime} \gg k_{\mathrm{f}}\right)$. In case (C) $\dot{R}$ vaporizes and does not participate in the reaction $\left(k_{\mathrm{f}} / v_{0}=k_{\mathrm{t}}=0\right)$.

For the kinetic scheme composed of eq 16-21, 23 and 24, the following equations hold with the steady-state assumption of each radical, i.e., of the total radical, $\dot{P}_{\mathrm{c}}, \dot{P}_{\mathrm{e}}$, and $\dot{R}$ for eq $42,43,44$, and 45 , respectively,

$$
\begin{aligned}
& R_{i}=k_{\mathrm{t}} \dot{P}+k_{\mathrm{t}} \dot{R} \quad\left(\dot{P}_{=}=\dot{P}_{\mathrm{c}}+\dot{P}_{\mathrm{e}}\right) \\
& R_{i}+\left(k_{\mathrm{f}} / v_{0}\right) \dot{P}_{\mathrm{e}}+\left(k_{\mathrm{f}} / v_{0}\right) \dot{R}=\left(k_{\beta}+k_{\mathrm{t}}\right) \dot{P}_{\mathrm{c}} \\
& k_{\beta} \dot{P}_{\mathrm{c}}-k_{\beta} \dot{P}_{\mathrm{c}} / N=\left(k_{\mathrm{t}}+k_{\mathrm{f}} / v_{0}\right) \dot{P}_{\mathrm{e}}+k_{\mathrm{d}} \dot{P}_{\mathrm{e}}^{1} \\
& k_{\mathrm{d}} \dot{P}_{\mathrm{e}}^{1}+k_{\beta} \dot{P}_{\mathrm{c}} / N=\left(k_{\mathrm{t}}+k_{\mathrm{f}} / v_{0}\right) \dot{R}
\end{aligned}
$$

where $N$ is the degree of polymerization and $\dot{P}_{\mathrm{e}}^{1}=r_{1} \dot{P}_{\mathrm{e}}$ is the amount of monomeric end radical. By solving eq $42-45$, the rate of weight loss, $-(\mathrm{d} M / \mathrm{d} t)$, the rate of chain scission, $-\mathrm{d} Z / \mathrm{d} t$, and the zip length, $\varepsilon=\mathrm{d} M / \mathrm{d} Z$, are expressed as,

$$
\begin{aligned}
-\mathrm{d} M / \mathrm{d} t & =k_{\mathrm{d}} \dot{P}_{\mathrm{e}}=k_{\mathrm{d}}\left\{k_{\beta}(1-1 / N) / F\right\} \dot{P} \\
& =\left\{k_{\mathrm{d}} k_{\beta}(1-1 / N) / F\right\} \cdot R_{i} /\left(k_{\mathrm{t}}+k_{\mathrm{t}}^{\prime} K\right)
\end{aligned}
$$

Table III. Zip length, $\varepsilon$, for the photoinitiated thermal degradation of PMMA with various initial molecular weights, $M_{n, 0}$, at $160^{\circ} \mathrm{C}$

\begin{tabular}{crr}
\hline$M_{n, 0}$ & $N_{0}$ & $\varepsilon$ \\
\hline $8.2 \times 10^{5}$ & 8400 & 1420 \\
$2.4 \times 10^{5}$ & 2400 & 325 \\
$1.27 \times 10^{5}$ & 1270 & 145 \\
$6.6 \times 10^{4}$ & 660 & 105 \\
\hline
\end{tabular}

$$
\begin{aligned}
\mathrm{d} Z / \mathrm{d} t & =k_{\beta} \dot{P}_{\mathrm{c}}=k_{\beta}\left\{\left(k_{\mathrm{t}}+k_{\mathrm{f}} / v_{0}+r_{1} k_{\mathrm{d}}\right) / F\right\} \cdot \dot{P} \\
& =\left\{k_{\beta}\left(k_{\mathrm{t}}+k_{\mathrm{f}} / v_{0}+r_{1} k_{\mathrm{d}}\right) / F\right\} \cdot R_{i} /\left(k_{\mathrm{t}}+k_{\mathrm{t}}^{\prime} K\right)
\end{aligned}
$$

$\varepsilon=\mathrm{d} M / \mathrm{d} Z=k_{\mathrm{d}}(1-1 / N) /\left(k_{\mathrm{t}}+k_{\mathrm{f}} / v_{0}+r_{1} k_{\mathrm{d}}\right)$

where

$$
\begin{gathered}
F=k_{\mathrm{t}}+k_{\mathrm{f}} / v_{0}+r_{1} k_{\mathrm{d}}+k_{\beta}(1-1 / N) \\
K=k_{\beta}\left\{r_{1} k_{\mathrm{d}}+\left(k_{\mathrm{t}}+k_{\mathrm{f}} / v_{0}\right) / N\right\} /\left\{F \cdot\left(k_{\mathrm{t}}^{\prime}+k_{\mathrm{f}}^{\prime} / v_{0}\right)\right\}
\end{gathered}
$$

and the fraction of monomeric end radical, $r_{1}=\dot{P}_{\mathrm{e}}^{1} / \dot{P}_{\mathrm{e}}$, is a function of $N$ as will be shown later.

Since the zip length, $\varepsilon$, determined experimentally depends on $N$ (Table III), we can conclude that in the denominator of eq 48 , the dominant factor is $r_{1} k_{\mathrm{d}}$, i.e.,

$$
r_{1} k_{\mathrm{d}} \gg k_{\mathrm{t}}+k_{\mathrm{f}} / v_{0}
$$

and

$$
\varepsilon=\mathrm{d} M / \mathrm{d} Z \cong 1 / r_{1}
$$

This holds for all the three cases.

With inequality 51 together with a reasonable relationship

$$
k_{\beta} \cong k_{\mathrm{d}} \gg r_{1} k_{\mathrm{d}},
$$

eq 46 is reduced to eq 54 ,

$$
\mathrm{d} M / \mathrm{d} t=k_{\mathrm{d}} \dot{P}_{\mathrm{e}}=k_{\mathrm{d}} R_{i} /\left[k_{\mathrm{t}}+\left\{k_{\mathrm{t}}^{\prime} r_{1} k_{\mathrm{d}} /\left(k_{\mathrm{t}}^{\prime}+k_{\mathrm{f}}^{\prime} / v_{0}\right\}\right]\right.
$$

In case $(\mathrm{A})$, using the relations 51 and 53 together with $k_{\mathrm{f}}^{\prime}=k_{\mathrm{f}}$ and $k_{\mathrm{t}}^{\prime}=k_{\mathrm{t}}$, eq 54 is reduced to eq 55, which shows the $N$ dependence of $-\mathrm{d} M / \mathrm{d} t\left(r_{1}\right.$ depends on $\left.N\right)$. For 
$k_{\mathrm{f}}^{\prime}=k_{\mathrm{f}}$ and $k_{\mathrm{t}}^{\prime}>k_{\mathrm{t}}$, we also get the $N$ dependence of $-\mathrm{d} M / \mathrm{d} t$.

$$
-\mathrm{d} M / \mathrm{d} t=\left(k_{\mathrm{d}} k_{\beta} / F\right) \dot{P} \cong\left(k_{\mathrm{t}}+k_{\mathrm{f}} / v_{0}\right) R_{i} /\left(k_{\mathrm{t}} r_{1}\right)
$$

In case (B) where the transfer of $\dot{R}$ is very fast, inequality 56 is reasonable.

$$
k_{\mathrm{f}}^{\prime} / v_{0} \gg k_{\mathrm{t}}^{\prime} \text { and } k_{\mathrm{t}}^{\prime} r_{1} k_{\mathrm{d}} / k_{\mathrm{t}}
$$

Then eq 54 is approximated by eq 57 , which is independent of degree of polymerization, $N$.

$$
-\mathrm{d} M / \mathrm{d} t \cong\left(k_{\mathrm{d}} / k_{\mathrm{t}}\right) R_{i}
$$

In case (C) where $\dot{R}$ vaporizes, eq 58 holds in place of eq 42 together with $k_{\mathrm{f}}^{\prime} / v_{0}=k_{\mathrm{t}}^{\prime}=0$.

$$
\begin{aligned}
R_{i} & =k_{\mathrm{t}} \dot{P}+k_{\mathrm{d}} r_{1} \dot{P}_{\mathrm{e}} \\
& =\left\{k_{\mathrm{t}}+k_{\mathrm{d}} r_{1} k_{\beta} /\left(k_{\mathrm{t}}+k_{\mathrm{f}} / v_{0}+r_{1} k_{\mathrm{d}}+k_{\beta}\right)\right\} \dot{P}
\end{aligned}
$$

By using inequalities 51 and 53 , eq 58 gives eq 59 , which shows the rate of weight loss,

$$
\begin{aligned}
-\mathrm{d} M / \mathrm{d} t & =k_{\mathrm{d}} \dot{P}_{\mathrm{e}}=\left\{k_{\mathrm{d}} k_{\beta} /\left(k_{\mathrm{t}}+k_{\mathrm{f}} / v_{0}+r_{1} k_{\mathrm{d}}+k_{\beta}\right)\right\} \dot{P} \\
& \cong R_{i} / r_{1}
\end{aligned}
$$

should depend on $N$.

The influence of the behavior of terminal radical, $\dot{R}$, on the $r_{1}$ (and hence $N$ ) dependences of zip length, $\mathrm{d} M / \mathrm{d} Z$, and rate of weight loss, $\mathrm{d} M / \mathrm{d} t$, are summarized in Table IV.

Thus, case (B) together with the condition of inequality 55 is consistent with the experimental results of $-\mathrm{d} M / \mathrm{d} t$ independent of $N$ and $\mathrm{d} M / \mathrm{d} Z$ depending of $N$ (Figure 13 and Table III). Cases (A) and (C) introduced $N$ dependence not only into $\mathrm{d} M / \mathrm{d} Z$ but also $-\mathrm{d} M / \mathrm{d} t$. Case (B) with inequalities 51,53 , and 55 also gives eq 60 , suggesting that $\mathrm{d} Z / \mathrm{d} t$ depends on $N$,

$$
\mathrm{d} Z / \mathrm{d} t \cong\left(r_{1} k_{\mathrm{d}} / k_{\mathrm{t}}\right) R_{i}
$$

which is consistent with Figure 14. The rapid chain transfer of terminal radical, $\dot{R}$, to polymer chain is supposed to be one of the important characteristics of thermal degradation of PMMA.

In order to obtain an explicit expression of the dependence of $r_{1}=\dot{P}_{\mathrm{e}}^{1} / \dot{P}_{\mathrm{e}}$ on $N$, the
Table IV. Influence of the behavior of the terminal radical, $R$, on the $N$ dependence of zip length and rate of weight loss
(A)
(B)
(C)

\begin{tabular}{cccc}
\hline $\begin{array}{c}\text { Behavior } \\
\text { of } \dot{R}\end{array}$ & $\begin{array}{c}\text { equal reactivity } \\
\text { to } \dot{P}_{\mathrm{e}}\end{array}$ & large $k_{\mathrm{f}}^{\prime}$ & Vaporization \\
\hline$\frac{\mathrm{d} M}{\mathrm{~d} Z} \cong$ & $1 / r_{1}$ & $1 / r_{1}$ & $1 / r_{1}$ \\
$\frac{\mathrm{d} M}{\mathrm{~d} t} \cong$ & $R_{i}\left(k_{\mathrm{t}}+k_{\mathrm{f}} / v_{0}\right) / k_{\mathrm{t}} r_{1}$ & $R_{i} k_{\mathrm{d}} / k_{\mathrm{t}}$ & $R_{i} / r_{1}$ \\
\hline
\end{tabular}

steady-state assumption for each $i$-mer endradical, $\dot{P}_{\mathrm{e}}^{i},(i=1 \sim N-1)$ was formulated as in eq 61 in place of eq 44

$$
k_{\beta} \dot{P}_{\mathrm{c}} / N=\left(k_{\mathrm{d}}+k_{\mathrm{f}} / v_{0}+k_{\mathrm{t}}\right) \dot{P}_{\mathrm{e}}^{N-1}
$$

$k_{\mathrm{d}} \dot{P}_{\mathrm{e}}^{N-1}+k_{\beta} \dot{P}_{\mathrm{c}} / N=\left(k_{\mathrm{d}}+k_{\mathrm{f}} / v_{0}+k_{\mathrm{t}}\right) \dot{P}_{\mathrm{e}}^{N-2}$

$$
k_{\mathrm{d}} \dot{P}_{\mathrm{e}}^{2}+k_{\beta} \dot{P}_{\mathrm{c}} / N=\left(k_{\mathrm{d}}+k_{\mathrm{f}} / v_{0}+k_{\mathrm{t}}\right) \dot{P}_{\mathrm{e}}^{1}
$$

using parameters $\alpha, \beta, \delta, \delta^{\prime}$ as

$$
\begin{aligned}
& \alpha=k_{\beta} \dot{P}_{\mathrm{c}} / k_{\mathrm{d}} N \\
& \beta=1+k_{\mathrm{f}} / k_{\mathrm{d}} v_{0}+k_{\mathrm{t}} / k_{\mathrm{d}}=1+\delta \quad(\delta<1) \\
& \delta^{\prime}=k_{\mathrm{f}}^{\prime} / k_{\mathrm{d}} v_{0}+k_{\mathrm{t}}^{\prime} / k_{\mathrm{d}}
\end{aligned}
$$

the amount of monomer end radical, $\dot{P}_{\mathrm{e}}^{1}$, the total amount of chain-end radical, $\dot{P}_{\mathrm{e}}$, and the amount of terminal radical, $\dot{R}$, are given by

$$
\begin{aligned}
\dot{P}_{\mathrm{e}}^{1}=(\alpha / \beta)\left(1+1 / \beta+\cdots+1 / \beta^{N-2}\right) & =\{\alpha /(\beta-1)\}\left(1-1 / \beta^{N-1}\right) \\
\dot{P}_{\mathrm{e}}= & \sum_{i=1}^{N-1} \dot{P}_{\mathrm{e}}^{i}=\{\alpha /(\beta-1)\}\{(1-1 / \beta) \\
& +\left(1-1 / \beta^{2}\right)+\left(1-1 / \beta^{3}\right)+\cdots \\
& \left.+\left(1-1 / \beta^{N-1}\right)\right\} \\
= & \left\{\alpha /(\beta-1)^{2}\right\}\{(N-1)(\beta-1) \\
& \left.-\left(1-1 / \beta^{N-1}\right)\right\} \\
\dot{R}= & \left(\dot{P}_{\mathrm{e}}^{1}+\alpha\right) / \delta^{\prime} \\
= & \left\{\alpha /(\beta-1) \delta^{\prime}\right\}\left\{\left(1-1 / \beta^{N-1}\right)+(\beta-1)\right\}
\end{aligned}
$$

Then $r_{1}$ can be approximated as, 


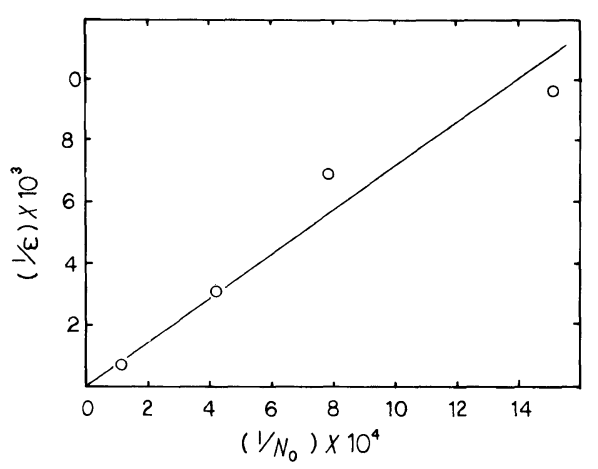

Figure 15. Plots of reciprocal zip length, $1 / \varepsilon$, against the reciprocal initial degree of polymerization, $1 / N_{0}$, for the photoinitiated thermal degradation of PMMA.

$$
\begin{aligned}
r_{1} & =\dot{P}_{\mathrm{e}}^{1} / \dot{P}_{\mathrm{e}}=\frac{\delta\left\{1-1 /(1+\delta)^{N-1}\right\}}{(N-1) \delta-\left\{1-1 /(1+\delta)^{N-1}\right\}} \\
& \cong \frac{2}{N}\left(1-\frac{N-2}{6} \delta+\cdots\right)
\end{aligned}
$$

and hence,

$$
1 / r_{1} \cong(N / 2)[1+\{(N-2) / 6\} \delta+\cdots]
$$

Equation 69 suggests that zip length, $\varepsilon=\mathrm{d} M /$ $\mathrm{d} Z \cong 1 / r_{1}$ is approximately proportional to degree of polymerization, $N$, as evident in Table III. The combination of eq 69 with eq 48 gives

$$
1 / \varepsilon \cong 2(1 / N)+\delta=(2 / \gamma)\left(1 / N_{0}\right)+\delta
$$

where $\gamma=N / N_{0}$ is the ratio of average degree of polymerization during degradation to its initial value. Plots of $1 / \varepsilon$ against $1 / N_{0}$ in Figure 15 give almost a straight line with an intercept at the origin, suggesting $\delta<10^{-4}$. This fact ascertains the inequality 51 . The slope in Figure 14 gives $\gamma=0.28$ which would be probable in the present experimental conditions.

In conclusion, the temperature range of chain scission for degradation of poly(methyl methacrylate) can be lowered from $270-330^{\circ} \mathrm{C}$ to the temperature range lower than $180^{\circ} \mathrm{C}$ by the photoinitiation method, and several kinetic parameters as well as their activation energies can be obtained. The number of main-chain scissions during degradation was calculated based on a effect of weight loss due to depolymerization. The low initiator efficiency for the formation of PMMA on-chain radical followed by the $\beta$-scission, depolymerization with zip length of $790-3200$, and first-order termination is the characteristics of photoinitated thermal degradation of poly(methyl methacrylate) with $N_{0} / 2 \geqq v$ at $140-180^{\circ} \mathrm{C}$. The molecular weight dependence of the photoinitiated thermal degradation of PMMA including the polymers with $N_{0} / 2<v$ revealed the mechanism including the first-order termination of polymer radical and rapid chain transfer to terminal radical, $\dot{R}$, to polymer chain.

Acknowledgments. This work was supported in part by a Grand-in-Aid on Special Project Research for “Energy" (No. 59040050) from the Ministry of Education, Science, and Culture of Japan.

\section{REFERENCES}

1. H. H. G. Jellinek, "Degradation of Vinyl Polymer," Academic Press, New York, 1951, p 74.

2. S. I. Madorsky, "Thermal Degradation of Organic Polymer," Interscience, New York, 1964.

3. I. Mita, in "Aspects of Degradation and Stabilization of Polymer," H. H. G. Jellinek, Ed., Elsever, Amsterdam, 1978, p 47.

4. (a) G. D. Fallon, M. L. Felsbourg, K. A. Holland, and I. D. Rae, Aust. J. Chem., 40, 501 (1987).

(b) K. A. Holland and I. D. Rae, Aust. J. Chem., 40, 687 (1987).

5. N. Grassie and J. R. MacCallum, J. Polym. Sci., B, 1, 551 (1963).

6. H. H. G. Jellinek and M. D. Luh, J. Phys. Chem., 70, 3672 (1968).

7. H. H. G. Jellinek and M. D. Luh, Makromol. Chem., 115, 89 (1968).

8. G. Bagby, R. Lehrle, and J. C. Robb, Makromol. Chem., 119, 122 (1968).

9. T. Kashiwagi, T. Hirata, and J. F. Brown, Macromolecules, 18, 131 (1985).

10. I. Mita, T. Hisano, K. Horie, and A. Okamoto, Macromolecules, 21, 3003 (1988).

11. K. Horie, K. Morishita, and I. Mita, Macromolecules, 17, 1746 (1984).

12. A. Salmassi and W. Schnabel, Polym. Photochem., 5, 215 (1984). 
13. P. R. E. J. Cowley and H. W. Melville, Proc. Roy. Soc. (London), Ser. A, 210, 461; ibid., 211, 320 (1952).
14. T. Hirata, T. Kashiwagi and J. E. Brown, Macromolecules, 18, 1410 (1985). 